\title{
Analyzing Density Operator in Thermal State for Complicated Time-Dependent Optical Systems
}

\author{
Jeong Ryeol Choi, ${ }^{1}$ Ji Nny Song, ${ }^{1}$ and Yeontaek Choi ${ }^{2}$ \\ ${ }^{1}$ Department of Radiologic Technology, Daegu Health College, Yeongsong-ro 15, Buk-gu, Daegu 702-722, Republic of Korea \\ ${ }^{2}$ Division of Mathematical Modeling, National Institute for Mathematical Sciences, KT Daeduk 2 Research Center, 463-1 Jeonmin-dong, \\ Yuseong-gu, Daejeon 305-390, Republic of Korea
}

Correspondence should be addressed to Jeong Ryeol Choi; choiardor@hanmail.net

Received 18 April 2014; Accepted 9 July 2014; Published 24 July 2014

Academic Editor: Kim Fook Lee

Copyright (C) 2014 Jeong Ryeol Choi et al. This is an open access article distributed under the Creative Commons Attribution License, which permits unrestricted use, distribution, and reproduction in any medium, provided the original work is properly cited.

Density operator of oscillatory optical systems with time-dependent parameters is analyzed. In this case, a system is described by a time-dependent Hamiltonian. Invariant operator theory is introduced in order to describe time-varying behavior of the system. Due to the time dependence of parameters, the frequency of oscillation, so-called a modified frequency of the system, is somewhat different from the natural frequency. In general, density operator of a time-dependent optical system is represented in terms of the modified frequency. We showed how to determine density operator of complicated time-dependent optical systems in thermal state. Usually, density operator description of quantum states is more general than the one described in terms of the state vector.

\section{Introduction}

Quantum behavior of time-dependent optical systems (TDOSs) is an interesting topic of study, that has attracted great concern in the literature of quantum physics for the last several decades [1-11]. As the knowledge of quantum mechanics deepen, the understanding of quantum characteristics of optical systems that have time-dependent parameters has become important as the counterpart of classical ones. Several methods for solving quantum solutions of TDOSs are known so far. They are invariant operator method [12], propagator method [13], and unitary or canonical transformation method [14]. Invariant operator method is the most common among them.

In traditional quantum mechanics, the state of a quantized system is described in terms of the state vector $|\psi\rangle$. However, if we use the density operator, the state can be described more generally. For instance, it is possible to treat the interaction between light and matter in terms of the density operator. The density operator of a system is obtained by making use of wave functions satisfying Schrödinger equation and can be used to derive various expectation values of physical quantities in thermal state.
The density operator will be described in thermal state using standard formalism relevant to invariant operator method. We study how to construct density operator in a general way by paying attention to the fact that density operator is represented in terms of the modified frequency of the system [15]. The density operator representation is a complete description of the information for a given system because it gives the knowledge that enables us to evaluate the outcome of any statistical measurement. If we consider that any quantum state regardless of being pure or mixed can be described by a single density matrix, the density operator is in particular useful for mixed states that in general take place from a statistical mixture of the initial states.

This paper is organized as follows. Preliminary quantum treatment of TDOSs is represented in Section 2 in connection with the density operator in thermal state. We see how to treat density operator of the system in more general way in Section 3, starting from the Liouville-von Neumann equation associated with the statistical density operator. We also apply our theory to several particular systems that have timedependent parameters. Finally, concluding remarks are given in Section 4. 


\section{Survey for a Simple Description of Density Operator}

Sometimes, parameters of an optical system may vary with time via its interaction with surroundings. In this case, the system is described by a time-dependent Hamiltonian. Let us consider a time-dependent Hamiltonian of the form

$$
\widehat{H}(\widehat{q}, \widehat{p}, t)=\frac{1}{2}\left[f(t) \widehat{p}^{2}+2 g(t)(\widehat{q} \widehat{p}+\widehat{p} \widehat{q})+h(t) \widehat{q}^{2}\right],
$$

where $f(t), g(t)$, and $h(t)$ are arbitrary real time functions. Not every classical solution of a system relevant to this Hamiltonian is oscillatory, but we assume that the optical system that we treat in this work is oscillatory for convenience. From Hamilton's equations, we see that the corresponding classical equation of motion is given by

$$
\begin{aligned}
& \frac{d^{2} q(t)}{d t^{2}}-\frac{\dot{f}(t)}{f(t)} \frac{d q(t)}{d t} \\
& \quad+\left(f(t) h(t)+\frac{2 \dot{f}(t) g(t)}{f(t)}-4 g^{2}(t)-2 \dot{g}(t)\right) q(t)=0 .
\end{aligned}
$$

It is known that quantum solutions of TDOSs are expressed in terms of the classical equations of motion. Since (2) is a second order differential equation, there are two linearly independent classical solutions for $q(t)$. Let us denote them by $s_{1}(t)$ and $s_{2}(t)$. When we develop quantum theory of the system, we put $s_{1}(t)$ and $s_{2}(t)$ as

$$
\begin{aligned}
& s_{1}(t)=s_{0} u(t), \\
& s_{2}(t)=s_{0} v(t),
\end{aligned}
$$

for the shake of convenience, where $s_{0}$ is a constant that has dimension of position and two time functions, $u(t)$ and $v(t)$, take no dimension.

To see the quantum features of TDOSs, it is useful to find a constant of motion (invariant operator). From $d \widehat{I} / d t=0$, we can easily derive a time constant associated with the system in the form [16]

$$
\widehat{I}=\hbar \Omega_{0}\left(\widehat{a}^{\dagger} \widehat{a}+\frac{1}{2}\right)
$$

where $\Omega_{0}$ is a Wronskian:

$$
\Omega_{0}=\frac{2}{f(t)}\left[s_{1}(t) \frac{d s_{2}(t)}{d t}-\frac{d s_{1}(t)}{d t} s_{2}(t)\right],
$$

and $\widehat{a}$ and $\widehat{a}^{\dagger}$ are annihilation and creation operators that are represented as

$$
\begin{aligned}
\widehat{a}= & \sqrt{\frac{1}{\hbar \Omega_{0}}} \\
& \times\left[\left(\frac{\Omega_{0}}{2 s(t)}-\frac{[2 g(t) s(t)-d s(t) / d t]}{i f(t)}\right) \hat{q}+i s(t) \hat{p}\right], \\
\hat{a}^{\dagger}= & \sqrt{\frac{1}{\hbar \Omega_{0}}} \\
& \times\left[\left(\frac{\Omega_{0}}{2 s(t)}+\frac{[2 g(t) s(t)-d s(t) / d t]}{i f(t)}\right) \hat{q}-i s(t) \hat{p}\right],
\end{aligned}
$$

with a time function of the form

$$
s(t)=\sqrt{s_{1}^{2}(t)+s_{2}^{2}(t)} .
$$

Notice that quantum wave functions $\psi_{n}(q, t)$ of TDOS, that satisfy Schrödinger equation, are represented in terms of the eigenstates $\phi_{n}(q, t)$ of $\widehat{I}$. In fact, it is well known that wave functions are given by $[1,2,12]$

$$
\psi_{n}(q, t)=\phi_{n}(q, t) e^{i \varphi_{n}(t)}
$$

where $\varphi_{n}(t)$ are some time-dependent phases. Once the eigenvalues $\phi_{n}(q, t)$ are obtained from the eigenvalue equation of $\widehat{I}, \varphi_{n}(t)$ are easily derived by solving Schrödinger equation after inserting (1) and (8) in it.

Quantum theory for describing the statistical state of optical systems requires the formalism of the density operator which is the quantum analogue of a phase space density given in classical statistical mechanics. From the Liouvillevon Neumann equation,

$$
\frac{\partial \widehat{\rho}(t)}{\partial t}+\frac{1}{i \hbar}[\hat{\rho}(t), \widehat{H}]=0,
$$

we can construct the density operator of the system. It is well known that the density operator of optical waves described with the simple harmonic oscillator (SHO) is represented in terms of Hamiltonian. However, for the case of TDOSs, the density operator is represented in terms of the invariant operator instead of the Hamiltonian. More clearly speaking, the Hamiltonian $\widehat{H}$ appearing in the density operator of SHO should be replaced with $y(0) \widehat{I}$, where $y(0)=f(0) /\left[2 s^{2}(0)\right]$. Hence, we can express the density operator of the system in the form $[2,15,16]$

$$
\widehat{\rho}(t)=\frac{1}{Z} e^{-\beta_{b} \hbar W\left(\widehat{a}^{\dagger} \widehat{a}+1 / 2\right)},
$$

where $Z$ is the partition function, $\beta_{b}=k_{b} T, k_{b}$ is Boltzmann's constant, and

$$
W=y(0) \Omega_{0} \text {. }
$$


This method of density operator formulation for Hamiltonian systems that have time-dependent parameters is firstly appeared in [17] as far as we know and afterwards extended to several quantum systems beyond optical ones [18-20]. The thermal state of the system is well described by the density operator given in (10) at least from the phenomenological point of view.

In fact $W$ is the same as the modified frequency of the system. For instance, for the standard damped harmonic oscillator with the natural angular frequency $\omega_{0}$ and the damping factor $\beta$, it becomes $W=\left[\omega_{0}^{2}-\beta^{2} / 4\right]^{1 / 2}(\equiv \omega)$ which is the exact modified frequency of the system (see Appendix A). In Fock state, the partition function is given by

$$
Z=\sum_{n=0}^{\infty}\left\langle\psi_{n}(t)\left|e^{-\beta_{b} \hbar W\left(\hat{a}^{\dagger} \widehat{a}+1 / 2\right)}\right| \psi_{n}(t)\right\rangle
$$

Because the density operator we introduced is Hermitian $\hat{\rho}^{\dagger}(t)=\hat{\rho}(t)$ as usual one for SHO, its eigenvalues are real. Equation (10) also obeys normalization condition, $\operatorname{Tr} \hat{\rho}(t)=$ 1. If the density operator is known, it is possible to obtain the expectation value of an arbitrary operator $\widehat{\mathcal{O}}(\widehat{q}, \widehat{p}, t)$ from $\langle\widehat{\mathcal{O}}(\widehat{q}, \widehat{p}, t)\rangle=\operatorname{Tr}[\widehat{\mathcal{O}}(\widehat{q}, \widehat{p}, t) \hat{\rho}(t)]$ as can be shown immediately from the properties of the trace connected to the element of density matrix. This is a fundamental consequence of quantum mechanics associated with an arbitrary measurement while the state vector is known only for a statistical sense.

\section{Generalized Formulation and Analysis}

Recall that, in the previous section, the density operator of the TDOS is represented in terms of $s(t)$. To treat the system more generally, we introduce a more common time function as $[21,22]$

$$
S(t)=\sqrt{c_{1} S_{1}^{2}(t)+c_{2} S_{1}(t) S_{2}(t)+c_{3} S_{2}^{2}(t)}
$$

where $c_{1}-c_{3}$ are arbitrary real constants and

$$
\begin{aligned}
& S_{1}(t)=s_{1,0} u(t), \\
& S_{2}(t)=s_{2,0} v(t) .
\end{aligned}
$$

If we put $s_{1,0}=s_{2,0} \equiv s_{0}, S_{1}(t)$ and $S_{2}(t)$ reduce to (3). Quantum problem of the system can be described in a more general way with the use of $S(t)$ instead of $s(t)$. Now the general quadratic invariant operator is given by

$$
\widehat{\mathscr{I}}=\hbar \Omega\left(\widehat{A}^{\dagger} \widehat{A}+\frac{1}{2}\right)
$$

where $\Omega$ is another Wronskian of the form

$$
\Omega=\frac{\sqrt{4 c_{1} c_{3}-c_{2}^{2}}}{f(t)}\left[S_{1}(t) \frac{d S_{2}(t)}{d t}-\frac{d S_{1}(t)}{d t} S_{2}(t)\right],
$$

and $\widehat{A}$ and $\widehat{A}^{\dagger}$ are generalized annihilation and creation operators:

$$
\begin{aligned}
\widehat{A}= & \sqrt{\frac{1}{\hbar \Omega}} \\
& \times\left[\left(\frac{\Omega}{2 S(t)}-\frac{[2 g(t) S(t)-d S(t) / d t]}{i f(t)}\right) \hat{q}+i S(t) \hat{p}\right], \\
\widehat{A}^{\dagger}= & \sqrt{\frac{1}{\hbar \Omega}} \\
& \times\left[\left(\frac{\Omega}{2 S(t)}+\frac{[2 g(t) S(t)-d S(t) / d t]}{i f(t)}\right) \hat{q}-i S(t) \hat{p}\right] .
\end{aligned}
$$

If we denote the eigenstates of $\widehat{\mathscr{I}}$ by $\Phi_{n}(q, t)$, the wave functions in Fock state can be represented as

$$
\Psi_{n}(q, t)=\Phi_{n}(q, t) e^{i \widetilde{\varphi}_{n}(t)}
$$

where $\widetilde{\varphi}_{n}(t)$ are another time-dependent phases. We can obtain $\widetilde{\varphi}_{n}(t)$ in a similar way to that of the previous case; that is, they can be obtained via some calculations after inserting (1) and (18) in Schrödinger equation.

As analogy to (11), if we define a time constant in this case as $\mathscr{W}^{\prime}=Y(0) \Omega$, where

$$
Y(0)=\frac{f(0)}{\left[2 S^{2}(0)\right]},
$$

$\mathscr{W}^{\prime}$ is somewhat different from the modified frequency of the system. Thus, in order to manage the density operator of the system, we introduce another time constant as

$$
\mathscr{W}=\kappa Y(0) \Omega,
$$

where $\kappa$ is a constant that should be determined later. Notice that $\kappa$ should be fixed in a way such that $\mathscr{W}$ become the modified frequency of the given system.

In terms of $\mathscr{W}$, we can represent the density operator in the form

$$
\widehat{\varrho}(t)=\frac{1}{\mathscr{Z}} e^{-\beta_{b} \hbar \mathscr{W}\left(\widehat{A}^{\dagger} \widehat{A}+1 / 2\right)},
$$

where $\mathscr{Z}$ is a new partition function. In Fock state, the partition function is given by

$$
\mathscr{Z}=\sum_{n=0}^{\infty}\left\langle\Psi_{n}(t)\left|e^{-\beta_{b} \hbar \mathscr{W}\left(\widehat{A}^{\dagger} \widehat{A}+1 / 2\right)}\right| \Psi_{n}(t)\right\rangle .
$$

Density operator contains all the physics and, as you can see here, has no phase in contrast to wave functions that are subject to the conventions of phase inherent in its constituent wave function. When an ensemble of states of the system is given, density operator is measurable in general. From this, we can infer its fundamental role as an object for describing the complete state of the (time-dependent) optical system. 
Now we will show how to determine $\kappa$ for particular systems with explicit choice of time functions in the Hamiltonian. Let us first choose the same time functions as those of the damped harmonic oscillator, that are given in Appendix A. Then, in this case, the solutions (14) are written as

$$
\begin{aligned}
& S_{1}(t)=s_{1,0} e^{-\beta t / 2} \cos (\omega t+\theta), \\
& S_{2}(t)=s_{2,0} e^{-\beta t / 2} \sin (\omega t+\theta) .
\end{aligned}
$$

By using (23), one can see that (20) becomes

$$
\mathscr{W}=\frac{1}{2} \kappa S \omega,
$$

where

$$
S=\frac{\sqrt{4 c_{1} c_{3}-c_{2}^{2}} s_{1,0} s_{2,0}}{c_{1} s_{1,0}^{2} \cos ^{2} \theta+c_{2} s_{1,0} s_{2,0} \cos \theta \sin \theta+c_{3} s_{2,0}^{2} \sin ^{2} \theta} .
$$

Hence, if we consider that $\mathscr{W}$ is the same as the modified frequency $\omega, \kappa$ is given by

$$
\kappa=\frac{2}{\mathrm{~S}} .
$$

We see that $\kappa$ is somewhat complicate for an arbitrary value of $\theta$.

In case of $\theta \rightarrow 0, \mathscr{W}$ becomes

$$
\mathscr{W}=\kappa \frac{\sqrt{4 c_{1} c_{3}-c_{2}^{2}} s_{2,0}}{2 c_{1} s_{1,0}} \omega .
$$

Hence, in this limit, $\kappa$ is given by

$$
\kappa=\frac{2 c_{1} s_{1,0}}{\sqrt{4 c_{1} c_{3}-c_{2}^{2}} s_{2,0}} .
$$

If we choose $c_{1}=c_{2}=1, c_{2}=0$, and $s_{1,0}=s_{2,0}$, (26) and (28) reduce to unity, leading to recovering the case of the previous section.

Let us consider another case that the Hamiltonian has the time function of the form

$$
f(t)=\frac{1}{m}, \quad g(t)=0, \quad h(t)=m \omega_{1}^{2}(t),
$$

where

$$
\omega_{1}(t)=\omega_{0}\left[1-\frac{\beta \omega_{0}}{\left[1+\beta \omega_{0} \cos ^{2}\left(\omega_{0} t\right)\right]^{2}}\right]^{1 / 2} .
$$

The classical solutions in this case are [23]

$$
\begin{aligned}
S_{1}(t)= & S_{1,0}\left[\frac{1}{\omega_{0}}+\beta \cos ^{2}\left(\omega_{0} t\right)\right]^{1 / 2} \\
& \times \cos \left(\int_{0}^{t} \frac{d t^{\prime}}{\left(1 / \omega_{0}\right)+\beta \cos ^{2}\left(\omega_{0} t^{\prime}\right)}+\theta\right), \\
S_{2}(t)= & S_{2,0}\left[\frac{1}{\omega_{0}}+\beta \cos ^{2}\left(\omega_{0} t\right)\right]^{1 / 2} \\
& \times \sin \left(\int_{0}^{t} \frac{d t^{\prime}}{\left(1 / \omega_{0}\right)+\beta \cos ^{2}\left(\omega_{0} t^{\prime}\right)}+\theta\right) .
\end{aligned}
$$

Using these, (20) is evaluated to be

$$
\mathscr{W}=\kappa \frac{\mathrm{S}}{2\left[\left(1 / \omega_{0}\right)+\beta\right]},
$$

where $S$ is the same as (25). Hence, by equating $\mathscr{W}$ with modified frequency of the system, $\kappa$ is given by

$$
\kappa=\frac{2\left[\left(1 / \omega_{0}\right)+\beta\right]}{\mathrm{S}} \times \text { Modified frequency. }
$$

Finally, we consider a more complicated case that the Hamiltonian has the time function of the form

$$
\begin{gathered}
f(t)=\frac{1}{m}\left(\frac{b}{t}\right)^{\alpha}, \quad g(t)=\frac{\beta}{t}, \\
h(t)=m\left(\frac{t}{b}\right)^{\alpha}\left(\omega_{0}^{2}+\frac{\gamma}{t^{2}}\right),
\end{gathered}
$$

where $b$ is a time scale and $\gamma$ is a constant. The corresponding classical equation of motion is

$$
\frac{d^{2} q(t)}{d t^{2}}+\frac{\alpha}{t} \frac{d q(t)}{d t}+\left(\omega_{0}^{2}+\frac{k}{t^{2}}\right) q(t)=0,
$$

where $k=\gamma-2 \alpha \beta-4 \beta^{2}+2 \beta$. The solutions of this equation are

$$
\begin{aligned}
& S_{1}(t)=s_{1,0} t^{(1-\alpha) / 2} J_{\sigma}\left(\omega_{0} t\right), \\
& S_{2}(t)=s_{2,0} t^{(1-\alpha) / 2} N_{\sigma}\left(\omega_{0} t\right),
\end{aligned}
$$

where $J_{\sigma}$ and $N_{\sigma}$ are the first and the second kind Bessel functions and $\sigma=\left(\left|1-4 k-2 \alpha+\alpha^{2}\right|\right)^{1 / 2} / 2$. Since $f(t)$ diverges as $t \rightarrow 0^{+}$, we replace $Y(0)$ with $Y(b)$; that is, $\mathscr{W}=\kappa Y(b) \Omega$. Then, we see that

$$
\mathscr{W}=\frac{\kappa t S^{\prime} \omega_{0}}{2 b},
$$

where

$$
\begin{aligned}
& S^{\prime}= \sqrt{4 c_{1} c_{3}-c_{2}^{2}} s_{1,0} s_{2,0} \eta(t) \\
& \times\left(2 \left[c_{1} s_{1,0}^{2} J_{\sigma}^{2}\left(\omega_{0} b\right)+c_{2} s_{1,0} s_{2,0} J_{\sigma}\left(\omega_{0} b\right) N_{\sigma}\left(\omega_{0} b\right)\right.\right. \\
&\left.\left.+c_{3} s_{2,0}^{2} N_{\sigma}^{2}\left(\omega_{0} b\right)\right]\right)^{-1},
\end{aligned}
$$

with

$$
\begin{aligned}
\eta(t)= & J_{\sigma}\left(\omega_{0} t\right)\left[N_{\sigma-1}\left(\omega_{0} t\right)-N_{\sigma+1}\left(\omega_{0} t\right)\right] \\
& +N_{\sigma}\left(\omega_{0} t\right)\left[J_{\sigma+1}\left(\omega_{0} t\right)-J_{\sigma-1}\left(\omega_{0} t\right)\right] .
\end{aligned}
$$

Through the same way as the previous cases, $\kappa$ is determined to be

$$
\kappa=\frac{2 b}{\omega_{0} S^{\prime} t} \times \text { Modified frequency. }
$$


The value of $\kappa$ is dependent on the choice of the form of $u$ and $v$. However, the physical amount of the quantity of the density operator is not affected by such choice. A somewhat different way to determine $\kappa$ in this case with another choice of $u$ and $v$ is shown in Appendix B.

From the choice of different time dependence of parameters, we can obtain different time evolutions of density operators with the different initial states. Density operator representation of a quantum optical system is equivalent to the wave function representation. However, there are many actual advantages of the use of density operators for quantum description of a certain TDOS, in particular, for the description of linear and nonlinear spectroscopy, thermal characteristics, relaxation in the condensed phase, and representation of photon states in time-varying media.

\section{Conclusion}

Density operator of a quantum TDOS is investigated in thermal state using invariant operator method. It is shown from Liouville-von Neumann equation for density operator that density operator is expressed in more general form, when the system contains time-dependent parameters, as given in (21). If we consider that quantum optical systems that undergo measurement or other similar quantum operations cannot be exclusively represented by wave functions, the statistical description in terms of density matrices is necessary.

We applied our theory to several examples of actual systems by choosing appropriate time functions. All calculations have been fulfilled within the theory of a quantized optical system with time-dependent parameters. As the system becomes complicate, the expression of the density operator is more or less intricate. On the other hand, for the case of optical wave described by the damped harmonic oscillator, the density operator reduces to the well known one [15] that is represented in terms of $\omega$.

\section{Appendices}

\section{A. Damped Harmonic Oscillator Limit}

Here, we show under the limit of standard damped harmonic oscillator that $W$ given in (11) becomes the modified frequency of the system. For the damped harmonic oscillator, the time functions of the Hamiltonian are given by

$$
f(t)=\frac{1}{m e^{\beta t}}, \quad g(t)=0, \quad h(t)=m e^{\beta t} \omega_{0}^{2},
$$

where $m, \beta$, and $\omega_{0}$ are constants. Then, the classical solutions (3) can be represented as

$$
\begin{aligned}
& s_{1}(t)=s_{0} e^{-\beta t / 2} \cos (\omega t+\theta), \\
& s_{2}(t)=s_{0} e^{-\beta t / 2} \sin (\omega t+\theta),
\end{aligned}
$$

where $\omega$ is a modified frequency of the form

$$
\omega=\left[\omega_{0}^{2}-\frac{\beta^{2}}{4}\right]^{1 / 2} \text {. }
$$

Now, using (5) and (11), we can easily show that [15]

$$
W=\omega,
$$

which corresponds to the exact modified frequency of the system.

\section{B. An Alternate Method}

We represent a somewhat different way for determining $\kappa$, which is necessary in the formulation of density operator, for a particular case that the time functions are given by (34). In this case, the classical solutions of the system can be reconstructed so that they involve sinusoidal function as

$$
\begin{aligned}
& S_{1}^{\prime}(t)=s_{1,0}^{\prime} t^{(1-\alpha) / 2} R_{\sigma}(t) \cos \left[\delta_{\sigma}(t)\right], \\
& S_{2}^{\prime}(t)=s_{2,0}^{\prime} t^{(1-\alpha) / 2} R_{\sigma}(t) \sin \left[\delta_{\sigma}(t)\right],
\end{aligned}
$$

where

$$
\begin{aligned}
& R_{\sigma}(t)=\left[J_{\sigma}^{2}\left(\omega_{0} t\right)+N_{\sigma}^{2}\left(\omega_{0} t\right)\right]^{1 / 2}, \\
& \delta_{\sigma}(t)=\tan ^{-1}\left[\frac{N_{\sigma}\left(\omega_{0} t\right)}{J_{\sigma}\left(\omega_{0} t\right)}\right] .
\end{aligned}
$$

Then, instead of (37), we have

$$
\mathscr{W}=\kappa \frac{t \omega_{0} S^{\prime \prime} R_{\sigma}^{2}(t)}{2 b R_{\sigma}^{2}(b)},
$$

where

$$
\begin{aligned}
S^{\prime \prime}= & \sqrt{4 c_{1} c_{3}-c_{2}^{2}} s_{1,0}^{\prime} s_{2,0}^{\prime} \\
\times & \left(c_{1} s_{1,0}^{\prime 2} \cos ^{2}\left[\delta_{\sigma}(b)\right]\right. \\
& +c_{2} s_{1,0}^{\prime} s_{2,0}^{\prime} \cos \left[\delta_{\sigma}(b)\right] \sin \left[\delta_{\sigma}(b)\right] \\
& \left.+c_{3} s_{2,0}^{\prime 2} \sin ^{2}\left[\delta_{\sigma}(b)\right]\right)^{-1} .
\end{aligned}
$$

Now, we can deduce $\kappa$ in the form

$$
\kappa=\frac{2 b R_{\sigma}^{2}(b)}{\omega_{0} R_{\sigma}^{2}(t) S^{\prime \prime} t} \times \text { Modified frequency. }
$$

\section{Conflict of Interests}

The authors declare that there is no conflict of interests regarding the publication of this paper.

\section{Acknowledgment}

This work was supported by the Basic Science Research Program through the National Research Foundation of Korea (NRF) funded by the Ministry of Education (Grant no. NRF2013R1A1A2062907). 


\section{References}

[1] J. R. Choi and S. Lyagushyn, "Nonclassical properties of superpositions of coherent and squeezed states for electromagnetic fields in time-varying media," in Quantum Optics and Laser Experiments, S. Lyagushyn, Ed., chapter 2, pp. 25-48, InTech, Rijeka, Croatia, 2012.

[2] J. R. Choi and A. Jamiolkowski, "Information theory and entropies for quantized optical waves in complex time-varying media," in Open Systems, Entanglement and Quantum Optics, chapter 6, pp. 121-138, InTech, Rijeka, Croatia, 2013.

[3] J. R. Choi, "Dissipative blackbody radiation: radiation in a lossy cavity," International Journal of Modern Physics B, vol. 18, no. 3, pp. 317-324, 2004.

[4] N. Ünal, "Quasi-coherent states for a photon in time varying dielectric media," Annals of Physics, vol. 327, no. 9, pp. 2177-2183, 2012.

[5] M. R. Bazrafkan and V. I. Man'ko, "Tomography of photonadded and photon-subtracted states," Journal of Optics B: Quantum and Semiclassical Optics, vol. 5, no. 4, pp. 357-363, 2003.

[6] A. B. Nassar, "New quantum squeezed states for the timedependent harmonic oscillator," Journal of Optics B: Quantum and Semiclassical Optics, vol. 4, no. 3, pp. S226-S228, 2002.

[7] S. K. Singh and S. Mandal, "The solutions of the generalized classical and quantum harmonic oscillators with time dependent mass, frequency, two-photon parameter and external force: the squeezing effects," Optics Communications, vol. 283, no. 23, pp. 4685-4695, 2010.

[8] A. G. Nerukh, T. M. Benson, and P. Sewell, "Influence on electromagnetic field of both time-varying medium in waveguide and its boundaries," in Proceedings of 6th International Conference on Transparent Optical Networks, pp. 156-160, July 2004.

[9] F. V. Fedotov, A. G. Nerukh, T. M. Benson, and P. Sewell, "Investigation of electromagnetic field in a layer with timevarying medium by Volterra integral equation method," Journal of Lightwave Technology, vol. 21, no. 1, pp. 305-314, 2003.

[10] B.-K. Chen, Y. Zhang, and B.-Q. Gao, "Solution of electromagnetic wave in time-varying media in two-dimensional space," Chinese Physics Letters, vol. 23, no. 3, pp. 595-598, 2006.

[11] N. V. Budko, "Electromagnetic radiation in a time-varying background medium," Physical Review A, vol. 80, no. 5, Article ID 053817, pp. 1-8, 2009.

[12] H. R. Lewis Jr. and W. B. Riesenfeld, "An exact quantum theory of the time-dependent harmonic oscillator and of a charged particle in a time-dependent electromagnetic field," Journal of Mathematical Physics, vol. 10, no. 8, pp. 1458-1473, 1969.

[13] J. H. Gweon and J. R. Choi, "Propagator and geometric phase of a general time-dependent harmonic oscillator," Journal of the Korean Physical Society, vol. 42, no. 3, pp. 325-330, 2003.

[14] J. R. Choi, B. J. Choi, and H. D. Kim, "Displacing, squeezing, and time evolution of quantum states for nanoelectronic circuits," Nanoscale Research Letters, vol. 8, article 30, pp. 1-13, 2013.

[15] J. R. Choi, M.-S. Kim, D. Kim, S. Menouar, and I. H. Nahm, "Information theories for time-dependent harmonic oscillator," Annals of Physics, vol. 326, no. 6, pp. 1381-1393, 2011.

[16] J. R. Choi, "Wigner distribution function for the timedependent quadratic-Hamiltonian quantum system using the Lewis-Riesenfeld invariant operator," International Journal of Theoretical Physics, vol. 44, no. 3, pp. 327-348, 2005.
[17] J.-Y. Ji and J. K. Kim, “Temperature changes and squeezing properties of the system of time-dependent harmonic oscillators," Physical Review A, vol. 53, no. 2, pp. 703-708, 1996.

[18] K. H. Cho, J. Y. Ji, S. P. Kim, C. H. Lee, and J. Y. Ryu, "Heisenbergpicture approach to the evolution of the scalar fields in an expanding universe," Physical Review D, vol. 56, no. 8, pp. 49164921, 1997.

[19] J. R. Choi, "Quantum and thermal state for exponentially damped harmonic oscillator with and without inverse quadratic potential," International Journal of Modern Physics B, vol. 16, no. 9, pp. 1341-1351, 2002.

[20] J. R. Choi and J. Y. Oh, "Thermal state for the capacitance coupled mesoscopic circuit with a power source," International Journal of Theoretical Physics, vol. 46, no. 7, pp. 1836-1852, 2007.

[21] C. J. Eliezer and A. Gray, "A note on the time-dependent harmonic oscillator," SIAM Journal on Applied Mathematics, vol. 30, no. 3, pp. 463-468, 1976.

[22] H. J. Korsch, “On Milne's quantum number function," Physics Letters A, vol. 109, no. 7, pp. 313-316, 1985.

[23] A. Angelow, "Frequency switching of quantum harmonic oscillator with time-dependent frequency," http://arxiv.org/pdf/ quant-ph/9803004. 

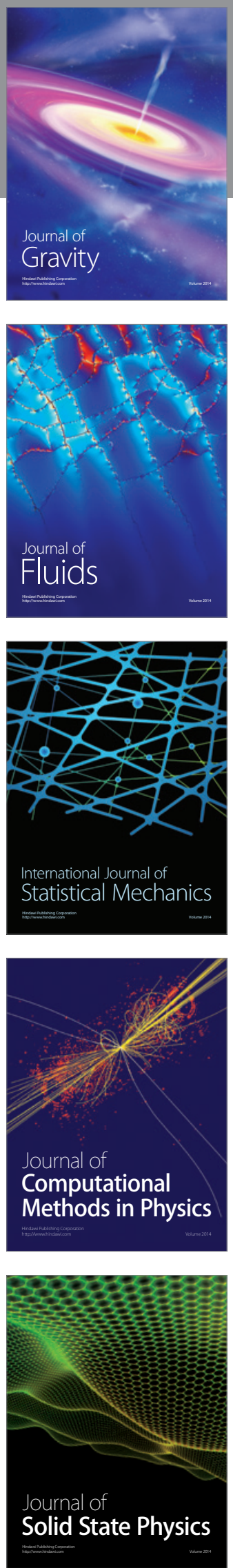

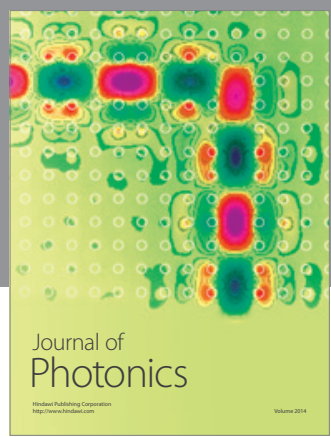

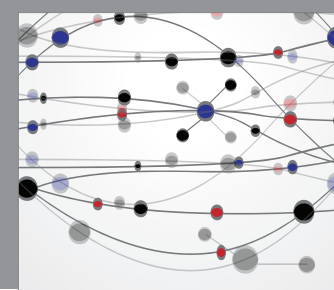

The Scientific World Journal

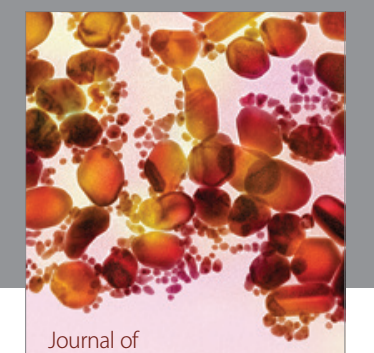

Soft Matter
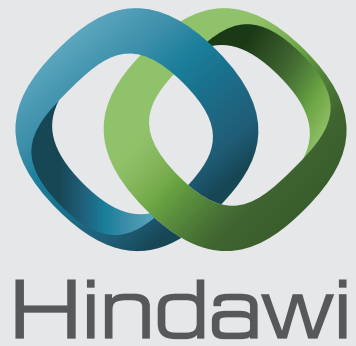

Submit your manuscripts at

http://www.hindawi.com
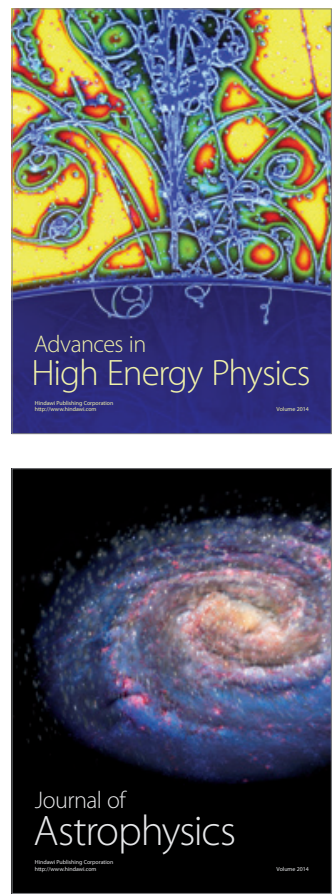
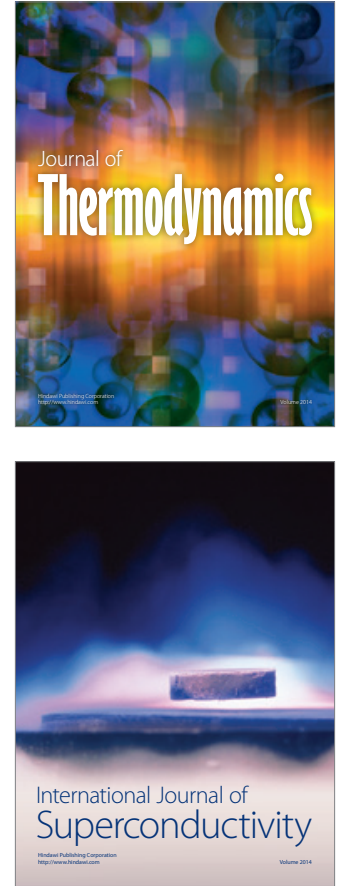
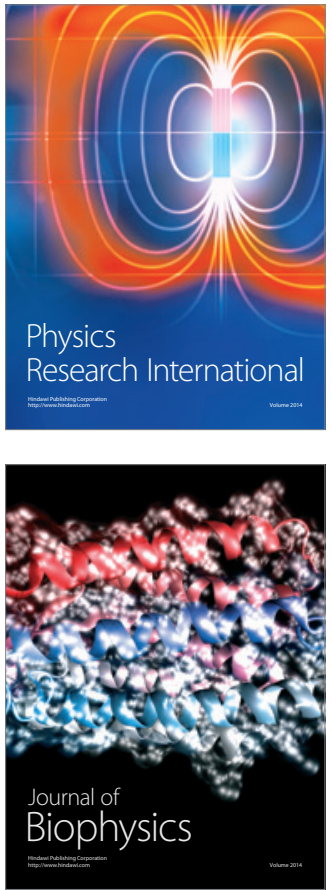
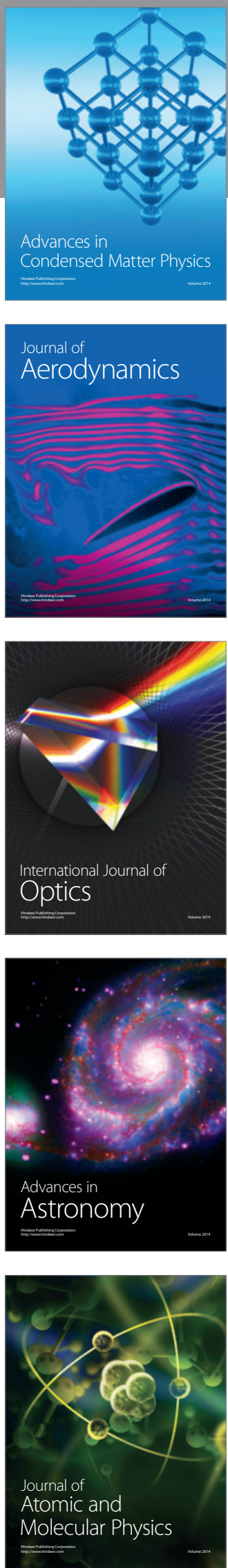